Proceedings of the $14^{\text {th }}$ Workshop on Adaptive and Reflective Middleware (ARM 2015)

Collocated with ACM/IFIP/USENIX Middleware 2015

December 8, 2015, Vancouver, Canada

Workshop Co-Chairs: Nikolaos Georgantas and Aniruddha Gokhale 


\section{Message from the Chairs}

Welcome to the Fourteenth Workshop on Adaptive and Reflective Middleware (ARM '15), which as in previous years is collocated with the ACM/IFIP/USENIX Middleware conference. Applying reflective techniques to middleware, and related software platforms for interoperability, one-to-many deployment, and adaptability, in order to "open up" their implementation, was explored in the previous workshops in this series and proved particularly successful and influential. Reflection by itself is today considered a baseline, yet it is insufficient to deliver the flexibility demanded by today's ever diversifying middleware environments, requiring higher and higher degrees of adaptability and resilience. This year's ARM workshop aims to follow on the success of previous editions by providing researchers with a forum to address this technological gap and explore how reflective approaches can be combined with complementary perspectives to support the complete life-cycle of highly adaptive middleware platforms.

This volume contains the papers presented at the workshop. ARM'15 attracted 11 submissions with authors from 11 different countries. Each submission was reviewed by at least three members of the program committee. Reviews were discussed electronically using the EasyChair conference management system. The Program Committee decided to accept 7 full papers max 6 pages each) and 3 short papers (max 3 pages each).

Putting together ARM '15 was a team effort. We would like to thank the authors for providing the content of the program, and the program committee for their hard work in reviewing the papers. We would also like to thank the ARM steering committee, specifically Nalini Venkatasubramanian, for their guidance. We are grateful to Middleware 2015 for allowing us to collocate our workshop with their conference from the inception of this workshop. Finally, we would like to thank ACM for their continued support of this workshop.

We hope that you will find this program inspiring and compelling, and that the conference will provide you with a valuable opportunity to share ideas with other researchers and practitioners from institutions around the world.

Nikolaos Georgantas and Aniruddha Gokhālé

ARM'15 Organizing Co-chairs 


\section{ARM 2015 Organization}

\section{Organizing Co-chairs}

Nikolaos Georgantas

Aniruddha Gokhālé

\section{Steering Committee}

Gordon Blair

Renato Cerqueira

Fabio M. Costa

Paulo Ferreira

Fabio Kon

Nalini Venkatasubramanian
Inria Paris-Rocquencourt, France

Vanderbilt University, USA
Lancaster University, UK

PUC-Rio, Brazil

Federal University of Goias, Brazil

INESC-ID, Portugal

University of Sao Paulo, Brazil

University of California, Irvine, USA

\section{Program Committee}

Kyoungho An

Anders Andersen

Paolo Bellavista

Gordon Blair

Yrom-David Bromberg

Renato Cerqueira

Fabio Costa

Edward Curry

Didier Donsez

Paulo Ferreira

Gang Huang

Fabio Kon

Thomas Ledoux

Philippe Merle

Sonia Ben Mokhtar

Romain Rouvoy

Douglas Schmidt

Alexandre Sztajnberg

Carolyn Talcott

Luís Veiga

Nalini Venkatasubramanian
Real-time Innovations, USA

UiT, The Arctic University of Norway, Norway

University of Bologna, Italy

Lancaster University, UK

LabRI - Université de Bordeaux, France

PUC-Rio, Brazil

Federal University of Goias, Brazil

Digital Enterprise Research Institute, Ireland

Université Joseph Fourier - Grenoble 1

Technical University of Lisbon, Portugal

Peking University, China

University of São Paulo, Brazil

Ecole des Mines de Nantes, France

INRIA, France

LIRIS CNRS, France

University of Lille / Inria, France

Vanderbilt University, USA

UERJ, Brazil

SRI International, USA

Universidade de Lisboa /INESC-ID, Portugal

University of California-Irvine, USA 


\section{Table of Contents}

\section{Preface}

1. Message from Workshop Co-Chairs

Nikolaos Georgantas and Aniruddha Gokhale

\section{Full Papers}

2. WF-Interop: Adaptive and Reflective REST Interfaces for Interoperability between Workflow Engines

Emad Heydari Beni, Bert Lagaisse, Wouter Joosen

3. SINk: A Middleware for Synchronization of Heterogeneous Software Interfaces

Mohammad Hosseini, Yu Jiang, Poliang Wu, Richard B. Berlin Jr., and Lui Sha

4. Adaptive middleware design for CPS: Considerations on the OS, resource managers, and the network run-time

Marisol García-Valls and Roberto Baldoni

5. Towards Energy-Proportional State-Machine Replication

Christopher Eibel and Tobias Distler

6. Holons: towards a systematic approach to composing systems of systems

Gordon Blair, Yérom-David Bromberg, Geoff Coulson, Yehia Elkhatib, Laurent Réveillère, Heverson B. Ribeiro, Etienne Rivière and François Taïani

7. On the suitability of black-box performance monitoring for SLA-driven cloud provisioning scenarios Arnaud Schoonjans, Dimitri Van Landuyt, Bert Lagaisse, Wouter Joosen

8. A Reflective Implementation of an Actor-based Concurrent Context-Oriented System Souhei Takeno and Takuo Watanabe

\section{Short Papers}

9. An Efficient Incremental Indexing Mechanism for Extracting Top-k Representative Queries Over Continuous Data-streams

Y.S.Horawalavithana and D.N.Ranasinghe

10. Dynamic Sets: A Programming Abstraction for Object Bundling

Matthias Prellwitz, Helge Parzyjegla and Gero Mühl

11. Decentralized view prediction for global content placement

Stéphane Delbruel, Davide Frey, and François Taïani 